Article

\title{
Serious Solutions for Unsteady Axisymmetric Flow over a Rotating Stretchable Disk with Deceleration
}

\author{
Muhammad Adil Sadiq \\ Department of Mathematics, DCC-KFUPM, KFUPM Box 5084, Dhahran 31261, Saudi Arabia; \\ adilsadiq@kfupm.edu.sa; Tel.: +966-13-868-3300
}

Received: 12 December 2019; Accepted: 2 January 2020; Published: 3 January 2020

check for updates

\begin{abstract}
In this article, the author has examined the unsteady flow over a rotating stretchable disk with deceleration. The highly nonlinear partial differential equations of viscous fluid are simplified by existing similarity transformation. Reduced nonlinear ordinary differential equations are solved by homotopy analysis method (HAM). The convergence of HAM solutions is also obtained. A comparison table between analytical solutions and numerical solutions is also presented. Finally, the results for useful parameters, i.e., disk stretching parameters and unsteadiness parameters, are found.
\end{abstract}

Keywords: homotopy analysis method; rotating stretchable disk; newtonian fluid; axisymmetric flow

\section{Introduction}

Recently, due to the massive practical application in the scientific and technical field, the study of the rotating stretchable disk has become significant, such as thermal power generation system, medical equipment's, computer storage devices, rotating machinery, gas turbine routers, air cleaning machines, crystal growth process, and in aerodynamic applications [1]. Initially, von Kármán [2] conducted a study on rotating disk. Several researchers then illustrated the different aspects of this important analysis. Fang and Zhang [3] have highlighted the flow between two stretchable disks and found the exact solutions. The parameters analysis and optimization of entropy generation in unsteady magneto hydrodynamics flow over a rotating stretchable flow over a rotating disk using artificial neural network and practical swarm optimization algorithm was presented by Rashidi et al. [4]. Recently, Fang and Tao [5] wrote about the unsteady flow over a rotating stretchable disk with deceleration. After using the similarity analysis, they found the numerical solutions.

In many situations, exact solutions are very difficult and in most of the cases exact solutions are impossible. Therefore, series solutions are more useful if they satisfy the given initial and boundary value problems. Nevertheless, there are various analytical approaches, and each approach has certain limitations. However, homotopy analysis method (HAM) has many advantages over many analytical methods. Liao [6] introduced the idea of HAM, which is used by many researchers effectively. Some useful studies are cited in [7-15]. The purpose of this article is to illustrate the application of HAM for unsteady Newtonian fluid flow over a rotating stretchable disk with declaration. Tables provide a correlation between current HAM solution and Fang and Tao's [5] numerical solution.

\section{Formulation of the Problem}

Let us consider an incompressible, laminar, and unsteady flow of a viscous fluid or Newtonian fluid over a stretchable disk, which is rotating about the $z$-axis with time dependent angular velocity $\Omega \prime(t)=\frac{\Omega}{1-b t}$, where $\Omega$ is constant angular speed of the disk and ' $b$ ' is the measure of unsteadiness. Flow is due to the rotation of the stretchable disk and is axisymmetric about the $z$-axis. Figure 1 describe 
the geometry of the proposed problem. The governing equations for an unsteady three-dimensional flow of viscous fluid in cylindrical coordinates are shown below.

$$
\begin{gathered}
\frac{1}{r} \frac{\partial}{\partial r}(r u)+\frac{\partial w}{\partial z}=0, \\
\frac{\partial u}{\partial t}+u \frac{\partial u}{\partial r}+w \frac{\partial u}{\partial z}-\frac{v^{2}}{r}=-\frac{1}{\rho} \frac{\partial p}{\partial r}+v\left(\frac{1}{r} \frac{\partial}{\partial r}\left(r \tau_{r r}\right)+\frac{\partial}{\partial z}\left(\tau_{z r}\right)-\frac{\tau_{\theta \theta}}{r}\right), \\
\frac{\partial v}{\partial t}+u \frac{\partial v}{\partial r}+w \frac{\partial v}{\partial z}-\frac{u v}{r}=v\left(\frac{\partial}{\partial r}\left(\tau_{r \theta}\right)+\frac{\partial}{\partial z}\left(\tau_{z \theta}\right)+\frac{\tau_{\theta r}+\tau_{r \theta}}{r}\right), \\
\frac{\partial w}{\partial t}+u \frac{\partial w}{\partial r}+w \frac{\partial w}{\partial z}=-\frac{1}{\rho} \frac{\partial p}{\partial z}+v\left(\frac{1}{r} \frac{\partial}{\partial r}\left(r \tau_{r z}\right)+\frac{\partial}{\partial z}\left(\tau_{z z}\right)\right),
\end{gathered}
$$

where $r$ is along the radial direction, $\theta$ is along the azimuthal direction, and $z$ is in normal direction to the axis. Here, Equation (1) is the continuity equation and Equations (2) and (4) represent the momentum equation for incompressible flow.

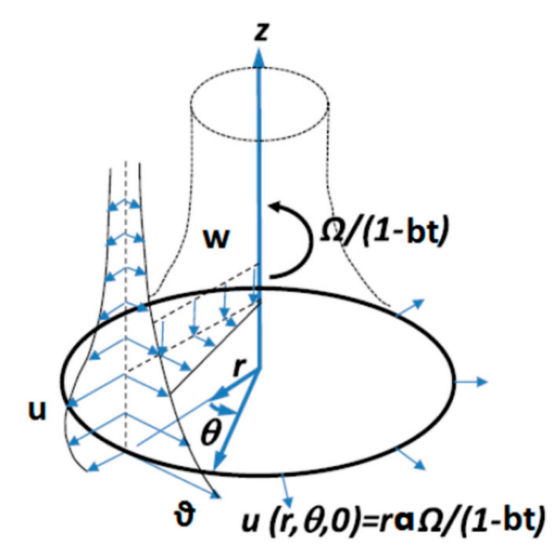

Figure 1. Geometry of the problem.

Where $u, v$, and $w$ are the velocities along $r, \theta$, and $z$ directions, $\rho$ is the density of fluid, $p$ is the pressure, $v$ is the kinematic viscosity, and $\tau_{r r}, \tau_{r \theta}, \tau_{z r}, \tau_{z \theta}, \tau_{z z}$ are the stress which are defined as

$$
\left[\begin{array}{ccc}
\tau_{r r} & \tau_{r \theta} & \tau_{r z} \\
\tau_{\theta r} & \tau_{\theta \theta} & \tau_{\theta z} \\
\tau_{z r} & \tau_{z \theta} & \tau_{z z}
\end{array}\right]=\left[\begin{array}{ccc}
2 \frac{\partial u}{\partial r} & \frac{\partial v}{\partial r}-\frac{v}{r} & \frac{\partial u}{\partial z}+\frac{\partial w}{\partial r} \\
\frac{\partial v}{\partial r}-\frac{v}{r} & \frac{2 u}{r} & \frac{\partial v}{\partial z} \\
\frac{\partial u}{\partial u}+\frac{\partial w}{\partial r} & \frac{\partial v}{\partial z} & 2 \frac{\partial w}{\partial z}
\end{array}\right]
$$

The proposed boundary conditions are specified in accordance with the geometry of the problem as

$$
\begin{gathered}
u(r, \theta, 0)=\frac{a \Omega r}{1-b t}, v(r, \theta, 0)=\frac{\Omega r}{1-b t}, w(r, \theta, 0)=0, \\
u(r, \theta, \infty)=v(r, \theta, \infty)=0,
\end{gathered}
$$

where ' $a$ ' is the disk stretching parameter.

Introducing, the similarity transformation used in [5] are

$$
\begin{gathered}
u=\frac{\Omega r}{1-b t} f^{\prime}(\eta), v=\frac{\Omega r}{1-b t} g(\eta), w=-2 \frac{\sqrt{\Omega v}}{\sqrt{1-b t}} f(\eta), \\
p=\frac{\rho v \Omega}{1-b t} P(\eta), \text { and } \eta=\sqrt{\frac{\Omega}{v}} \frac{z}{\sqrt{1-b t}} .
\end{gathered}
$$


Applying these similarities into the above equations, following non-dimensional equations along with boundary conditions can be obtained as

$$
\begin{gathered}
f^{\prime \prime \prime}+2 f f^{\prime \prime}-f^{\prime 2}+g^{2}=S\left(\frac{\eta}{2} f^{\prime \prime}+f^{\prime}\right), \\
g^{\prime \prime}-2 f^{\prime} g-2 f g^{\prime}=S\left(\frac{\eta}{2} g \prime+g\right), \\
P^{\prime}=2 f^{\prime \prime}+4 f f^{\prime \prime}-S\left(\eta f^{\prime}+f\right), \\
f(0)=0, f^{\prime}(0)=a, g(0)=1, f^{\prime} \rightarrow 0 \text { and } g \rightarrow 0, \text { as } \eta \rightarrow \infty,
\end{gathered}
$$

where $S=b / \Omega$ is the unsteadiness parameter.

\section{Homotopy Analysis Method}

Homotopy Analysis Method (HAM) [6-15] is used to find an analytical solution to Equations (8) and (11). The velocity distribution $f(\eta)$ and $g(\eta)$ can be expressed by a set of base functions

$$
\left\{\eta^{n} \exp { }^{(-m \eta)} \mid m, n \geq 0\right\}
$$

in the form

$$
\begin{aligned}
& f(\eta)=a_{0,0}^{0}+\sum_{n=0}^{\infty} \sum_{k=0}^{\infty} a_{m, n}^{k} \eta^{n} \exp ^{(-m \eta)}, \\
& g(\eta)=b_{0,0}^{0}+\sum_{n=0}^{\infty} \sum_{k=0}^{\infty} b_{m, n}^{k} \eta^{n} \exp ^{(-m \eta)}
\end{aligned}
$$

in which $a_{m, n}^{k}$ and $b_{m, n}^{k}$ are the coefficients, the initial guesses $f_{0}$ and $g_{0}$ can be selected on the basis of the law of the solution expressions and of the boundary conditions:

$$
\begin{gathered}
f_{0}(\eta)=a\left(1-\exp ^{-\eta}\right), \\
g_{0}(\eta)=\exp ^{-\eta} .
\end{gathered}
$$

The auxiliary linear operators are

$$
\begin{aligned}
& \mathcal{L}_{1}=\frac{d^{3}}{d \eta^{3}}+\frac{d^{2}}{d \eta^{2}} \\
& \mathcal{L}_{2}=\frac{d^{2}}{d \eta^{2}}+\frac{d}{d \eta}
\end{aligned}
$$

which satisfy

$$
\begin{gathered}
\mathcal{L}_{1}\left[C_{1}+C_{2} \exp ^{-\eta}+C_{3} \eta\right]=0, \\
\mathcal{L}_{2}\left[C_{4} \exp ^{-\eta}+C_{5}\right]=0,
\end{gathered}
$$

where $C_{i}(i=1-5)$ are integral constants.

\subsection{Zeroth-Order Deformation Equation}

If $q \in[0,1]$ denote an embedding parameter, $\hbar_{f}$ and $\hbar_{g}$ indicate the non zero auxiliary parameters for $f(\eta)$ and $g(\eta)$, the zeroth-order deformations for the given problem are

$$
\begin{aligned}
& (1-q)_{f}\left[\hat{f}(\eta ; q)-f_{0}(\eta)\right]=q \hbar_{f} N_{f}[\hat{f}(\eta ; q), \hat{g}(\eta ; q)], \\
& (1-q)_{g}\left[\hat{g}(\eta ; q)-g_{0}(\eta)\right]=q \hbar_{g} N_{g}[\hat{g}(\eta ; q), \hat{f}(\eta ; q)],
\end{aligned}
$$




$$
\hat{f}(0 ; q)=0, \hat{f}^{\prime}(0 ; q)=a, \hat{g}(0 ; q)=1 \hat{f}^{\prime}(\infty ; q)=\hat{g}(\infty ; q)=0 .
$$

Defining the nonlinear operators for the above problem as

$$
\begin{gathered}
N_{f}[\hat{f}(\eta ; q), \hat{g}(\eta ; q)]=\frac{\partial^{3} \hat{f}(\eta ; q)}{\partial \eta^{3}}+2 \hat{f}(\eta ; q) \frac{\partial^{2} \hat{f}(\eta ; q)}{\partial \eta^{2}}-\left(\frac{\partial \hat{f}(\eta ; q)}{\partial \eta}\right)^{2} \\
+(\hat{g}(\eta ; q))^{2}-S\left(\frac{\eta}{2} \frac{\partial^{2} \hat{f}(\eta ; q)}{\partial \eta^{2}}+\frac{\partial \hat{f}(\eta ; q)}{\partial \eta}\right), \\
N_{g}[\hat{g}(\eta ; q), \hat{f}(\eta ; q)]=\frac{\partial^{2} \hat{g}(\eta ; q)}{\partial \eta^{2}}+2 \hat{f}(\eta ; q) \frac{\partial \hat{g}(\eta ; q)}{\partial \eta} \\
-2 \hat{g}(\eta ; q) \frac{\partial \hat{f}(\eta ; q)}{\partial \eta}-s\left(\frac{\eta}{2} \frac{\partial \hat{g}(\eta ; q)}{\partial \eta}+\hat{g}(\eta ; q)\right) .
\end{gathered}
$$

For $q=0$ and $q=1$, one can have

$$
\begin{aligned}
& \hat{f}(\eta ; 0)=f_{0}(\eta), \hat{f}(\eta: 1)=f(\eta), \\
& \hat{g}(\eta ; 0)=g_{0}(\eta), \hat{g}(\eta: 1)=g(\eta) .
\end{aligned}
$$

By Taylor's theorem

$$
\begin{aligned}
& \hat{f}(\eta ; q)=f_{0}(\eta)+\sum_{m=1}^{\infty} f_{m}(\eta) q^{m}, f_{m}(\eta)=\left.\frac{1}{m !} \frac{\partial^{m} f(\eta ; q)}{\partial \eta^{m}}\right|_{q=0}, \\
& \hat{g}(\eta ; q)=g_{0}(\eta)+\sum_{m=1}^{\infty} g_{m}(\eta) q^{m}, g_{m}(\eta)=\left.\frac{1}{m !} \frac{\partial^{m} g(\eta ; q)}{\partial \eta^{m}}\right|_{q=0},
\end{aligned}
$$

and

$$
\begin{aligned}
& f(\eta)=f_{0}(\eta)+\sum_{m=1}^{\infty} f_{m}(\eta), \\
& g(\eta)=g_{0}(\eta)+\sum_{m=1}^{\infty} g_{m}(\eta) .
\end{aligned}
$$

\subsection{Mth-Order Deformation}

Differentiating the zeorth-order deformation Equations (21) and (23) with respect to $q$, then setting $q=0$, and finally dividing them by $m !$, the mth-order deformation equations can be obtained as

$$
\begin{gathered}
\mathcal{L}_{1}\left[f_{m}(\eta)-\chi_{m} f_{m-1}(\eta)\right]=\hbar_{f} R_{m}^{f}(\eta), \\
\mathcal{L}_{2}\left[g_{m}(\eta)-\chi_{m} g_{m-1}(\eta)\right]=\hbar_{g} R_{m}^{g}(\eta), \\
f_{m}(0)=f_{m}^{\prime}(0)=g_{m}(0)=f_{m}^{\prime}(\infty)=g_{m}(\infty)=0,
\end{gathered}
$$

where

$$
\begin{gathered}
R_{m}^{f}(\eta)=f_{m-1}^{\prime \prime \prime}+2 \sum_{k=0}^{m-1} f_{k} f_{m-1-k}^{\prime \prime}-\sum_{k=0}^{m-1}{f^{\prime}}_{k} f^{\prime}{ }_{m-1-k}+\sum_{k=0}^{m-1} g_{k} g_{m-1-k}-S\left(\frac{\eta}{2} f_{m-1}^{\prime \prime}+f^{\prime}{ }_{m-1}\right), \\
R_{m}^{g}(\eta)=g_{m-1}^{\prime \prime}-2 \sum_{k=0}^{m-1} g_{k} f^{\prime}{ }_{m-1-k}+2 \sum_{k=0}^{m-1} f^{\prime}{ }_{k} g^{\prime}{ }_{m-1-k}-S\left(\frac{\eta}{2} g_{m-1}^{\prime}+g_{m-1}\right), \\
\chi_{m}= \begin{cases}0, & m \leq 1, \\
1, & m>1,\end{cases}
\end{gathered}
$$


in which $f_{m}(\eta)$ and $g_{m}(\eta)$ denote the special solutions of Equations (32) and (33) and the $C_{i}(i=1-5)$ integral constants are calculated by the (34) boundary conditions. Equations (32) and (34) can be solved using Mathematica for $m=1,2,3 \ldots$.

\section{Convergence of the HAM Solution}

The homotopy analysis method includes the regulating parameter $h$, which controls the region of convergence and HAM solution approximation. To ensure that the solutions converge within the admissible spectrum of auxiliary parameter values and $h f$ and $h g, h-$ curves were sketched for 15th-order approximation. The $h-$ curves are plotted in Figures 2 and 3. The admissible ranges of values of $h f$ and $h g$ are $-1.5 \leq h_{f}<-0.3$ and $-1.5 \leq h_{g}<-0.3$, these ranges vary with the change in parameters.

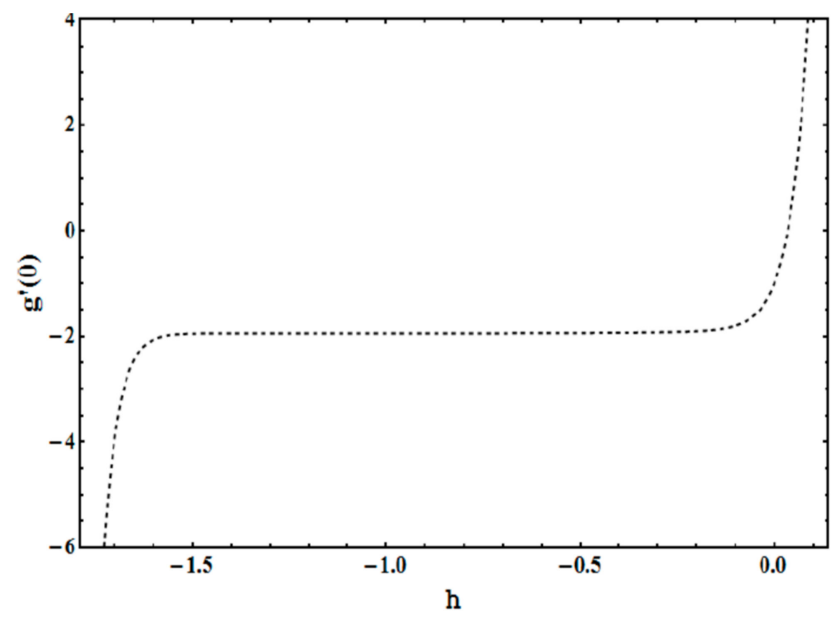

Figure 2. 15th-order $g^{\prime}(0)$ for $a=1$ and $S=-1$.

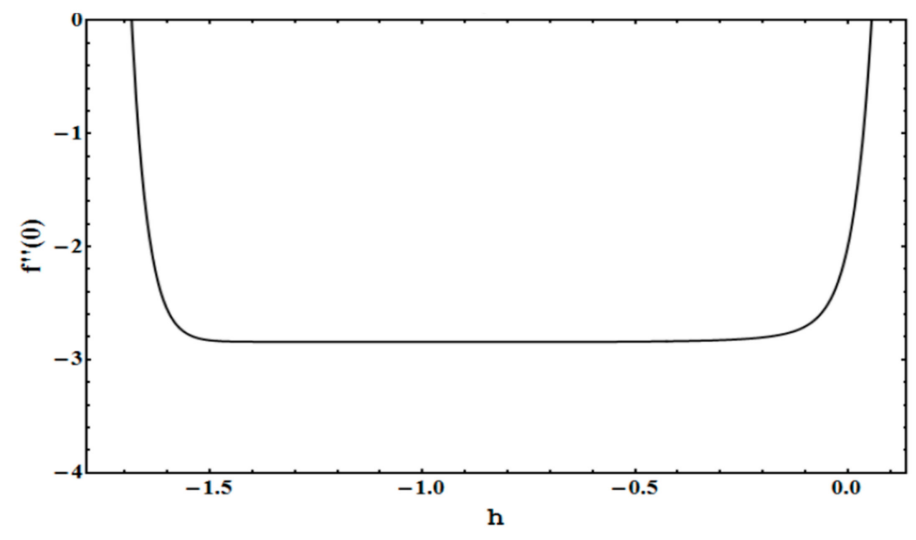

Figure 3. 15th-order $f^{\prime \prime}(0)$ for $a=1$ and $S=-1$.

\section{Results and Discussion}

To solve Equations (8) and (9) homotopy analysis method (HAM) is applied as a subject to the boundary conditions (11). Homotopy analysis method is a strong analytical technique which is applied to obtain the convergent series solution of nonlinear differential equations. The convergence region for HAM through $h$-curves are sketched and analyzed in Figures 3 and 4. Homotopy analysis method provides great freedom to obtain the convergent result. The convergence region varies for different values of $a$ and $S$. Tables 1-4 represent the convergence of solution for different values of parameters. The error analysis of the obtained approximated results is as follows.

$$
E_{m}=\int_{0}^{\infty} e_{m}^{2}(t) d t
$$


where $e_{m}(t)$ is the residual error of Equations (8) and (9) at the mth-order approximation. It is observed that 10th-order approximation is in good agreement with the numerical result.

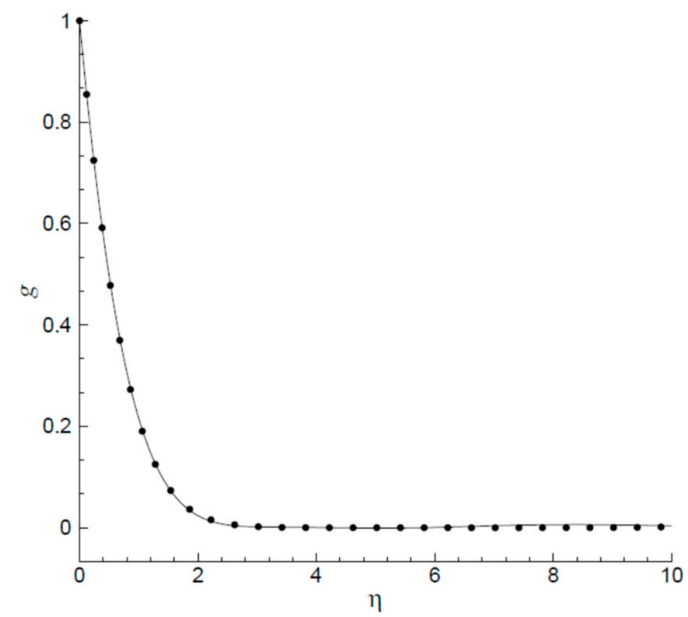

Figure 4. Comparison of convergence of $g(\eta)$ when $\hbar=-0.333, a=1$ and $S=-1$ (line: 10th-order, dots: 5th-order).

Table 1. Comparison of the numerical result [5] with homotopy analysis method (HAM) convergent result when $a=1, S=-1$ and $\hbar_{f}=-1 / 3, \hbar_{g}=-1 / 4$.

\begin{tabular}{ccccc}
\hline Order & $f^{\prime \prime}(0)$ & $g^{\prime}(0)$ & error $f$ & error $g$ \\
\hline 2nd & -0.7673 & -1.196 & 0.311 & 0.044 \\
4th & -0.6945 & -1.246 & 0.021 & 0.0067 \\
6th & -0.6681 & -1.264 & 0.0046 & 0.0021 \\
8th & -0.6581 & -1.270 & 0.00091 & 0.000715 \\
10th & -0.6543 & -1.271 & 0.00014 & 0.00023 \\
\hline \multicolumn{5}{c}{ Numerical result $[5] f^{\prime}(0)=-0.6520, g^{\prime}(0)=-1.2716}$.
\end{tabular}

Table 2. Comparison of the numerical result [5] with HAM convergent result when $a=1, \mathrm{~S}=-1 / 10$ and $\hbar_{f}=-1 / 3, \hbar_{g}=-1 / 4$.

\begin{tabular}{ccccc}
\hline Order & $f^{\prime \prime}(0)$ & $g^{\prime}(0)$ & error $f$ & error $g$ \\
\hline 2nd & -0.9642 & -1.3321 & 0.031 & 0.379 \\
4th & -0.9374 & -1.4162 & 0.0047 & 0.0055 \\
6th & -0.9262 & -1.446 & 0.00075 & 0.00091 \\
8th & -0.9217 & -1.458 & 0.00012 & 0.00016 \\
10th & -0.9200 & -1.4627 & 0.000018 & 0.000033 \\
\hline
\end{tabular}

Numerical result $[5] f^{\prime}(0)=-0.9189, g^{\prime}(0)=-1.4656$.

Table 3. Comparison of the numerical result [5] with HAM convergent result when $a=2, \mathrm{~S}=-1 / 10$ and $\hbar_{g}=\hbar_{f}=-1 / 5$.

\begin{tabular}{ccccc}
\hline Order & $f^{\prime \prime}(0)$ & $g^{\prime}(0)$ & error $f$ & error $g$ \\
\hline 2nd & -2.779 & -1.658 & 0.408 & 0.543 \\
4th & -2.9729 & -1.847 & 0.0876 & 0.136 \\
6th & -3.044 & -1.924 & 0.0234 & 0.0437 \\
8th & -3.072 & -1.953 & 0.0071 & 0.018 \\
10th & -3.082 & -1.958 & 0.0024 & 0.012 \\
\hline
\end{tabular}

Numerical result $[5] f^{\prime}(\mathbf{0})=-3.1178, g^{\prime}(0)=-2.0530$. 
Table 4. Comparison of the numerical result [5] with HAM convergent result when $a=1, S=-1 / 2$ and $\hbar_{g}=\hbar_{f}=-1 / 4$.

\begin{tabular}{ccccc}
\hline Order & $f^{\prime \prime}(0)$ & $g^{\prime}(0)$ & error $f$ & error $g$ \\
\hline 2nd & -0.9062 & -1.2760 & 0.1051 & 0.0221 \\
4th & -0.8592 & -1.3424 & 0.0283 & 0.0037 \\
6th & -0.8319 & -1.3654 & 0.0077 & 0.00082 \\
8th & -0.8172 & -1.3741 & 0.0021 & 0.00021 \\
10th & -0.8093 & -1.3774 & 0.00058 & 0.00006 \\
\hline \multicolumn{4}{c}{ Numerical result $[5] f^{\prime}(\mathbf{0})=-0.8007, g^{\prime}(0)=-1.3797}$.
\end{tabular}

The convergence control parameter plays an important role. In Tables 5 and 6 , the effect of $\hbar$ on convergence is shown. Tables 5 and 6 show that the convergence of the solution depends strongly on $\hbar$. It can be seen easily that for one set of $\hbar$ the convergence is faster than the other.

Table 5. The Convergence analysis of $f^{\prime \prime}(0)$ for different $\hbar$ when $a=1$, and $S=-1 / 2$.

\begin{tabular}{ccccc}
\hline Order & $\hbar f=-1 / 4, \hbar g=-1 / 5$ & Err & $\hbar f=-1 / 5, \hbar g=-1 / 4$ & Err \\
\hline 2nd & -0.9007 & 0.1062 & -0.9243 & 0.1405 \\
4th & -0.8535 & 0.0283 & -0.8826 & 0.0514 \\
6th & -0.8282 & 0.0076 & -0.8658 & 0.0312 \\
8th & -0.8149 & 0.0021 & -0.8325 & 0.0069 \\
10th & -0.8080 & 0.0005 & -0.8201 & 0.0025 \\
\hline \multicolumn{4}{c}{ Numerical result [5] $\boldsymbol{f}^{\prime}(\mathbf{0})=-0.8007}$.
\end{tabular}

Table 6. The Convergence analysis of $g^{\prime}(0)$ for different $\hbar$ when $a=1$, and $S=-1 / 2$.

\begin{tabular}{ccccc}
\hline Order & $\hbar f=-1 / 4, \hbar g=-1 / 5$ & Err & $\hbar f=-1 / 5, \hbar g=-1 / 4$ & Err \\
\hline 2nd & -1.2345 & 0.03718 & -1.2786 & 0.022 \\
4th & -1.3157 & 0.0087 & -1.3445 & 0.0036 \\
6th & -1.3502 & 0.0024 & -1.3578 & 0.0016 \\
8th & -1.366 & 0.0007 & -1.3737 & 0.00021 \\
10th & -1.3734 & 0.0002 & -1.3768 & 0.00006 \\
\hline
\end{tabular}

Numerical result $[5] g^{\prime}(\mathbf{0})=1.3797$.

In Figures 5 and 6, the comparison of 5th-order approximation with 10th-order approximation is shown, which again provide the facts for convergence. The Mathematica software is used to compute the results for higher-order approximation. As the given problem is highly nonlinear, the computation time increases if higher-order approximation is computed or increases the value of the parameters. For $a=1$ and $S=0$, the given problem becomes a special case as mentioned in the numerical paper [5]. Table 7 provides the convergence result for this special case as well. The results obtained in the present research for this special case are also in very good agreement with the numerical result. This shows the strength of homotopy analysis methods. It is found that for small $S, f^{\prime \prime}(0)$ decreases with the increase of ' $a$ ' as shown in Tables 2 and 3. Figure 7 represents the velocity distribution for different values of $a$. It is observed that with the increase in disk stretching parameter the velocity decreases. Figures 8 and 9 show that with the decrease in the unsteadiness parameter, both tangential and radial velocities increase. 


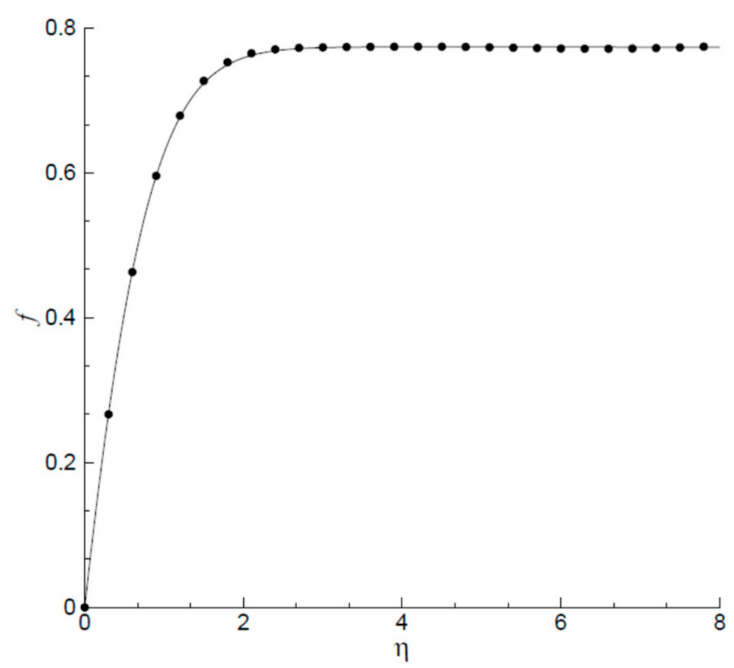

Figure 5. Comparison of convergence of $f(\eta)$ when $\hbar=-0.333, a=1$ and $S=-1$ (line: 10th-order, dots: 5th-order).

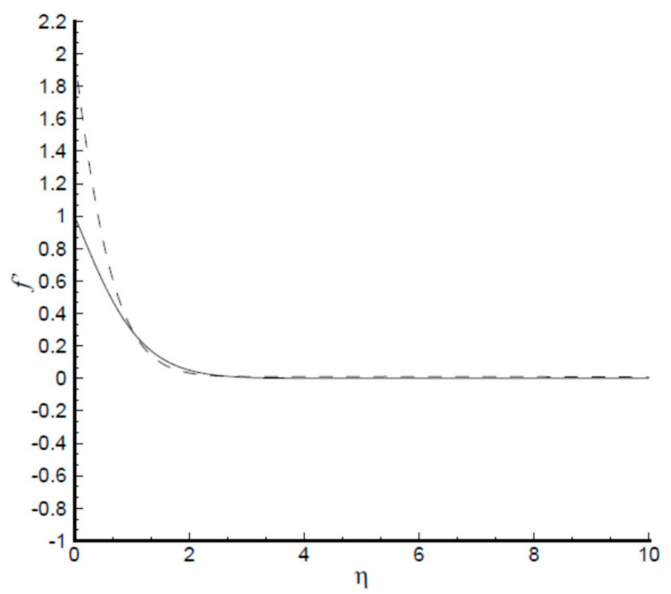

Figure 6. For $S=-1 / 2$ solid line: $a=1$, Dashed line: $a=2$ 10th order HAM approximation for $f^{\prime}(\eta)$.

Table 7. Comparison of the numerical result [5] with HAM convergent result for special case when analysis $=1, S=0$, and $\hbar f=-28 / 100, \hbar g=-1 / 3$.

\begin{tabular}{ccccc}
\hline Order & $f^{\prime \prime}$ & $g^{\prime}$ & error $f$ & error $g$ \\
\hline 5th & -1.1785 & -1.44639 & 0.00044518 & 0.00019581 \\
10th & -1.1751 & -1.45359 & 0.00001437 & $8.13 \times 10^{-7}$ \\
15th & -1.1739 & -1.45402 & $3.86 \times 10^{-7}$ & $1.15 \times 10^{-8}$ \\
20th & -1.1737 & -1.45406 & $2.02 \times 10^{-8}$ & $6.42 \times 10^{-10}$ \\
\hline \multicolumn{5}{c}{ Numerical result [5] $f^{\prime \prime}(0)=-1.1737, g^{\prime}(0)=-1.4541}$.
\end{tabular}




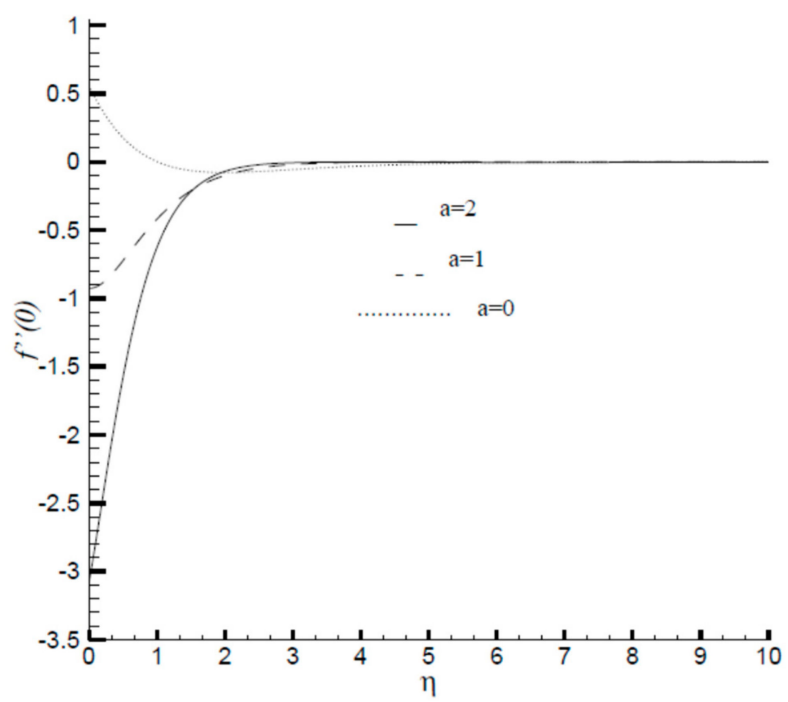

Figure 7. For $S=-1 / 10$ 10th-order HAM approximation for $f^{\prime \prime}(\eta)$.

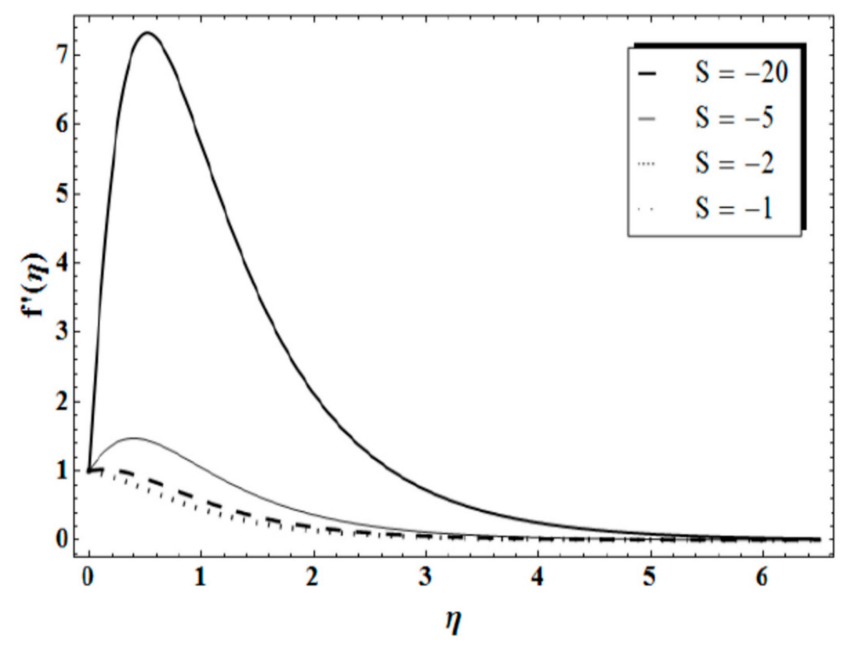

Figure 8. Variation of $f^{\prime}(\eta)$ for different values of unsteadiness parameter for $a=1$.

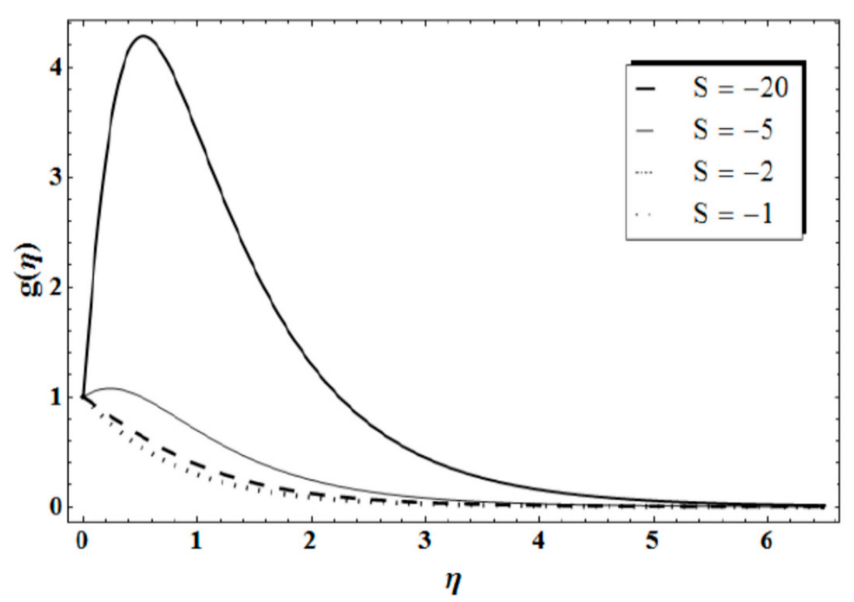

Figure 9. Variation of $g(\eta)$ for different values of unsteadiness parameter for $a=1$.

\section{Conclusions}

In this research, viscous axisymmetric flow is studied on a stretchable rotating disk with deceleration. It is found that Navier-Stokes equation admits a similarity solution, which depends on 
non-dimensionalized parameters $S$ and $a$ measuring unsteadiness and disk stretching, respectively. The resulting group of nonlinear ordinary differential equations is then solved analytically using homotopy analysis method (HAM). In numerical paper [5] it is mentioned that there are two solution branches. The upper solution branch is physically feasible, but the lower solution branch may not be practically possible. Here, the author has discussed and evaluated the outcome for a physical solution from the upper field.

The main results are summarized as

- Results obtained by homotopy analysis method are in good agreement with existing numerical results;

- All the velocity profiles decrease with an increase in unsteadiness parameter $S$;

- Radial and axial velocity of the flow increases with the increase in disk stretching parameter $a$, whereas tangential velocity shows a decreasing trend with an increase in $a$;

- Variation trend decays with faster velocity to the ambient for fast deceleration as compared to the slow deceleration of the disk.

Funding: This research received funding from KFUPM.

Acknowledgments: The author wishes to express his thanks for the support received from King Fahd University of Petroleum and Minerals.

Conflicts of Interest: Author declare no conflicts of interest with any one.

\section{References}

1. Turkyilmazoglu, M. Three-dimensional MHD stagnation flow due to a stretchable rotating disk. Int. J. Heat Mass Transf. 2012, 55, 6959-6965. [CrossRef]

2. Von Kármán, T. Über Laminar Und Turbulente Reibung. J. Appl. Math. Mech. 1921, 1, 233-252.

3. Fang, T.; Zhang, J. Flow between two stretchable disks-An exact solution of the NavierStokes equations. Int. Commun. Heat Mass Transf. 2008, 35, 892-895. [CrossRef]

4. Rashidi, M.M.; Ali, M.; Freidoonimehr, N.; Nazari, F. Parametric analysis and optimization of entropy generation in unsteady MHD flow over a stretching rotating disk using articial neural network and particle swarm optimization algorithm. Energy 2013, 55, 1-14. [CrossRef]

5. Fang, T.; Hua, T. Unsteady viscous flow over a rotating stretchable disk with deceleration. Commun. Nonlinear Sci. Numer. Simul. 2012, 17, 5064-5072. [CrossRef]

6. Liao, S. On the homotopy analysis method for nonlinear problems. Appl. Math. Comput. 2004, 147, 499-513. [CrossRef]

7. Nadeem, S.; Awais, M. Thin film flow of an unsteady shrinking sheet through porous medium with variable viscosity. Phys. Lett. A 2008, 372, 4965-4972. [CrossRef]

8. Nadeem, S.; Ali, M. Analytical solutions for pipe flow of a fourth grade fluid with Reynold and Vogel's models of viscosities. Commun. Nonlinear Sci. Numer. Simul. 2009, 14, 2073-2090. [CrossRef]

9. Nadeem, S.; Abbasbandy, S.; Hussain, M. Series solutions of boundary layer flow of a Micropolar fluid near the stagnation point towards a shrinking sheet. Z. Fur Nat. 2009, 64, 575-582. [CrossRef]

10. Nadeem, S. Thin film flow of a third grade fluid with variable viscosity. Z. Fur Nat. 2009, 64, 553-558. [CrossRef]

11. Nadeem, S.; Hussain, A. MHD flow of a viscous fluid on a non-linear porous shrinking sheet by Homotopy analysis method. Appl. Math. Mech. 2009, 30, 1569-1578. [CrossRef]

12. Khan, H.; Ram, N.M.; Vajravelu, K.; Liao, S.J. The explicit Series Solution of SIR and SIS Epidemic Models. Appl. Math. Comput. 2009, 215, 653-669. [CrossRef]

13. Khan, H.; Liao, S.J.; Ram, N.M.; Vajravelu, K. An analytical solution for a nonlinear time delay model in Biology. Commun. Nonlinear Sci. Numer. Simul. 2009, 14, 3141-3148. [CrossRef]

14. Khan, H.; Xu, H. Series Solution of Thomas Fermi Atom Model. Phys. Lett. A 2007, 365, 111-115. [CrossRef]

15. Liao, S. A short review on the homotopy analysis method in fluid mechanics. J. Hydrodyn. 2010, 22, 882-884. [CrossRef] 
(C) 2020 by the author. Licensee MDPI, Basel, Switzerland. This article is an open access article distributed under the terms and conditions of the Creative Commons Attribution (CC BY) license (http://creativecommons.org/licenses/by/4.0/). 